\title{
Predominance of Late-Spiking Neurons in Layer VI of Rat Perirhinal Cortex
}

\author{
John P. McGann, ${ }^{1}$ James R. Moyer Jr, ${ }^{2}$ and Thomas H. Brown ${ }^{1,2,3}$ \\ 1/nterdepartmental Neuroscience Program and Departments of ${ }^{2}$ Psychology and ${ }^{3}$ Cellular and Molecular Physiology, Yale \\ University, New Haven, Connecticut 06520
}

Recent work demonstrated the importance of perirhinal cortex $(\mathrm{PR})$ in a variety of behavioral tasks and disease processes. Studies from our laboratory revealed that some layers of PR contain neurons with unusual properties. Here we report a detailed examination of the cellular neurobiology of layer $\mathrm{VI}$ of $\mathrm{PR}$, using whole-cell recordings and biocytin cell fills in horizontal rat brain slices. The most striking finding is that an overwhelming majority ( $\sim 86 \%$ ) of neurons are late-spiking (LS) cells, which can delay the onset of their spike trains by several seconds or more relative to the onset of a depolarizing current step. LS neurons previously have been shown to exist only in very small numbers in a limited number of other cortical regions. Anatomical reconstructions have revealed that the LS neurons vary greatly in morphology, including both pyramidal and nonpyramidal cells. Another surprising physiological finding is the fact that single-spiking (SS) neurons are the second most common cell type ( $7 \%)$. SS neurons issue only a single action potential even in response to extreme depolarization. They have been seen previously in the amygdala, but never in cortex. A third remarkable finding is that there are almost no regular spiking (RS) neurons, unlike all other cortical regions that have been studied. This unique abundance of LS neurons in layer $\mathrm{VI}$, along with the presence of SS neurons and the absence of RS neurons, demonstrates that layer $\mathrm{VI}$ of PR is unlike any other cortical region that has been studied to date.

Key words: perirhinal; amygdala; late spiking; temporal cortex; slowly inactivating; timing; classical conditioning; entorhinal; morphology; delay; pyramidal; nonpyramidal
The perirhinal cortex (PR) of the rat receives polymodal sensory input and makes reciprocal connections with the amygdala, entorhinal cortex, and frontal cortex, placing it in a critical anatomical position for a number of behavioral tasks (Suzuki, 1996; Burwell and Amaral, 1998a,b). Lesions of PR have been shown to impair performance on object recognition and delayed nonmatch-tosample (DNMS) tasks in humans (Buffalo et al., 1998), monkeys (Meunier et al., 1993), and rats (Otto and Eichenbaum, 1992; Mumby and Pinel, 1994; Wiig and Burwell, 1998), especially when the subject is required to store stimulus information for $>1 \mathrm{sec}$ or so. Similar lesions in rats have been shown to disrupt fear conditioning, where the time interval between the conditioned stimulus onset and the unconditioned stimulus commonly spans several seconds or more (Falls et al., 1997).

Behavioral findings encourage the thought (Otto and Eichenbaum, 1992) that PR may play a special role in certain temporal aspects of information processing or short-term storage. A natural question is whether there might be anything special about the cellular neurobiology that would suggest some unusual differences in the time domain. Surprisingly, nothing was known about the cellular neurobiology until very recently. Our first general survey suggested that PR is indeed unusual with respect to the frequency distribution of various cell types across layers

\footnotetext{
Received Nov. 15, 2000; revised April 23, 2001; accepted April 26, 2001.

This work was supported by National Institutes of Health Grants RO1 48660 and RO1 50948 (T.H.B.) and by a predoctoral fellowship from the National Science Foundation (J.P.M.). We thank Sharon Furtak, Tyrone Powell, and Britt Payne for assistance with the morphology and Ewan McNay for assistance with data collection.

Correspondence should be addressed to Dr. Thomas H. Brown, Department of Psychology, P.O. Box 208205, Yale University, New Haven, CT 06520. E-mail: thomas.brown@yale.edu.

Copyright (C) 2001 Society for Neuroscience $\quad 0270-6474 / 01 / 214969-08 \$ 15.00 / 0$
}

(Faulkner and Brown, 1999). The impression from this initial survey prompted a more thorough layer-by-layer analysis.

Here we present data on the cellular neurophysiology and neuroanatomy of layer VI of PR. The results differ greatly from our findings in layer II/III (Beggs et al., 2000) and layer V (J. Moyer, E. McNay, and T. Brown, unpublished observations) of PR. Moreover, the frequency distribution of cell types in layer VI is unlike any other cortical region that has been studied thus far. Our cellular results reinforce an emerging speculation (Otto and Eichenbaum, 1992; Tieu et al., 1999; McCreless et al., 2000; McGann and Brown, 2000) that PR may be specialized for some aspect of information processing that occurs on a seconds range time scale.

\section{MATERIALS AND METHODS}

Slice preparation. A majority of brain slices $(90 \%)$ were prepared from young male Sprague Dawley rats aged 10-22 d old (mean, 15.3; SD, 2.8). Horizontal brain slices through perirhinal cortex and the lateral nucleus of the amygdala were prepared as described previously (Moyer and Brown, 1998). Briefly, rats were anesthetized deeply with halothane and decapitated; the brains were removed quickly and placed in ice-cold oxygenated "cutting" saline [composition (in mM) 206 sucrose, 2-2.8 $\mathrm{KCl}, 1 \mathrm{CaCl}_{2}, 1 \mathrm{MgCl}_{2}, 2 \mathrm{MgSO}_{4}, 1.25 \mathrm{NaH}_{2} \mathrm{PO}_{4}, 26 \mathrm{NaHCO}_{3}, 10$ D-glucose] for $\sim 3 \mathrm{~min}$. Each brain was blocked, hemisected, and glued ventral surface up to the tray of a temperature-controlled vibratome. Horizontal brain slices $400 \mu \mathrm{M}$ thick through PR at the level of the lateral nucleus of the amygdala were cut at $\sim 1^{\circ} \mathrm{C}$. Slices were placed into individual wells of our 24-well slice incubation chamber (Moyer and Brown, 1998). The chamber contained our standard oxygenated recording saline [composition (in mM) $124 \mathrm{NaCl}, 2-2.8 \mathrm{KCl}, 2 \mathrm{CaCl}_{2}, 2 \mathrm{MgSO}_{4}$, $1.25 \mathrm{NaH}_{2} \mathrm{PO}_{4}, 26 \mathrm{NaHCO}_{3}$, and $10 \mathrm{D}$-glucose, $\left.\mathrm{pH} 7.4,295 \mathrm{mOsm}\right]$. This solution also was used to perfuse slices continuously during recording. Slices were allowed to recover at room temperature $\left(23-25^{\circ} \mathrm{C}\right)$ for at least $60 \mathrm{~min}$ before any experiments were begun.

In a minority of experiments $(10 \%)$ we used adult male Sprague Dawley rats (104-107 d old; 438-469 gm). These slices were prepared 
identically to those from young animals. However, in some experiments the slices were incubated at $\sim 35^{\circ} \mathrm{C}$ for 30 min immediately after sectioning, after which they were incubated at room temperature. This technique is used in many labs because it facilitates obtaining whole-cell recordings in adult brain slices (Colbert and Johnston, 1996; Moyer and Brown, 1998).

Whole-cell recording techniques and locations. An upright microscope (Zeiss Axioskop, Oberkochen, Germany) equipped with a $63 \times$ water immersion objective ( 0.90 numerical aperture) or a $40 \times$ water immersion lens (0.75 numerical aperture), infrared filtered light with differential interference contrast optics (IR-DIC), and a Hamamatsu C2400 video camera and video enhancement device were used to visualize neurons and patch electrodes (Moyer and Brown, 1998). In some experiments, video images were captured on an Apple Macintosh Quadra 950, using NIH Image software (Bethesda, MD) and a frame grabber card.

Perirhinal cortex was defined according to the coordinates of Burwell and colleagues (1995). The slices that were used corresponded to plates 98 through 100 ( -3.8 to -5.2 relative to bregma) of the rat stereotaxic atlas of Paxinos and Watson (1998). In this study we recorded only from the part of PR directly adjacent to the lateral nucleus of the amygdala. The external capsule was plainly visible under high power, and we used it as a landmark in selecting cells specifically located in layer VI of PR. Electrode placement in layer V I was confirmed under low power for most cells and was established additionally from the cell fills. The depth of each cell within the slice was measured after most recordings (mean, 87.7 $\mu \mathrm{m}$; SD, $19.5 \mu \mathrm{m})$.

Whole-cell recordings were made from 86 visually identified neurons in layer VI of PR by using an EPC-7 amplifier (List-Medical) or an AxoPatch 1D amplifier (Axon Instruments, Foster City, CA) as described previously (Moyer and Brown, 1998). Only cells with a stable resting potential more negative than $-73 \mathrm{mV}$ and with overshooting action potentials of at least $60 \mathrm{mV}$ (measured from threshold) were included in the present study. All recordings were made from the cell body with room temperature $\left(23-24^{\circ} \mathrm{C}\right)$ recording saline, except for a small number of experiments performed at $30-32^{\circ} \mathrm{C}$. Patch electrodes were fabricated from borosilicate glass (inner diameter, $1.303 \mathrm{~mm}$; outer diameter, $1.689 \mathrm{~mm}$; Drummond Scientific, Broomall, PA) on a Sutter Instruments P-87 or P-97 puller (Novato, CA). Pipettes were filled with the following solution (in $\mathrm{mM}$ ): $110 \mathrm{~K}$-gluconate, $10 \mathrm{HEPES}$, 1.0 EGTA, $20 \mathrm{KCl}, 2.0 \mathrm{MgCl}_{2}, 2.0 \mathrm{Na}_{2} \cdot \mathrm{ATP}$, and $0.3 \mathrm{Na}_{2} \cdot \mathrm{GTP}, \mathrm{pH}$ 7.3, 290 mOsm. In most experiments the electrodes also contained $0.5 \%$ biocytin to permit subsequent visualization of the neuronal morphology. In some experiments the electrode tips were fire polished on a Narishige microforge (East Meadow, NY).

Signals were filtered at 3 and $10 \mathrm{kHz}$ (EPC-7 amplifier) or at $2 \mathrm{kHz}$ (AxoPatch 1D amplifier), digitized at $44 \mathrm{kHz}$, and stored on VCR tape with a Neurocorder (Neurodata Instruments). In most experiments the data also were digitized on-line, using an Instrutech ITC-16 (Great Neck, NY), and acquired in real time, using Axodata (Axon Instruments) or custom acquisition software written for IgorPro (WaveMetrics, Lake Oswego, OR). In the remaining experiments this was done during off-line playback from the tape.

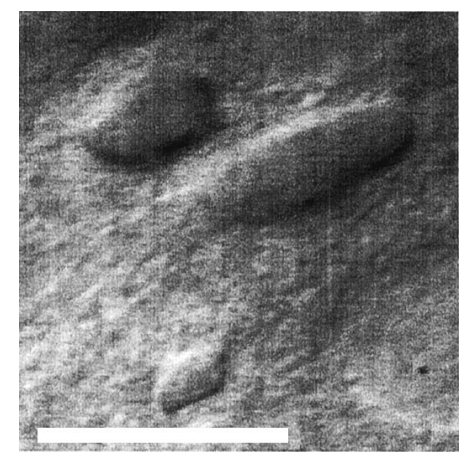

Figure 1. Video image of neurons in layer VI of perirhinal cortex. This is an infrared differential interference contrast image of PR layer VI of the sort we used to select neurons for recording. The small neuron near the bottom of the figure has the morphology of a fast-spiking neuron, which is infrequent in this region. The two larger neurons are typical layer VI nonpyramidal neurons. Scale bar, $25 \mu \mathrm{m}$.
Electrode resistances were measured on the basis of the response to a $5 \mathrm{mV}$ hyperpolarizing voltage step with the electrode in the bath (mean electrode resistance, $2.9 \pm 0.1 \mathrm{M} \Omega$ ). Gigaohm seal resistances were measured on the basis of the response to a $25 \mathrm{mV}$ hyperpolarizing voltage step in cell-attached patch configuration (mean seal resistance, $5.6 \pm 0.4 \mathrm{G} \Omega$ ). Resting potential was measured as soon as the membrane potential was stable after whole-cell break-in (typically within a few minutes). Because a vast majority of cells we recorded from were latespiking cells, we used 5-sec-long alternating hyperpolarizing and depolarizing current steps to study their membrane properties. For each cell the amplitude of the current step was increased in increments of 1-5 pA near rheobase to study the latency of action potential onset at threshold. In a few experiments longer current steps of $10 \mathrm{sec}$ or more also were given to investigate the duration of sustained firing. In a subset of experiments a small $(2-10 \mathrm{pA})$ hyperpolarizing current step was given repeatedly to assist in computing the time constant of membrane voltage response near rest.

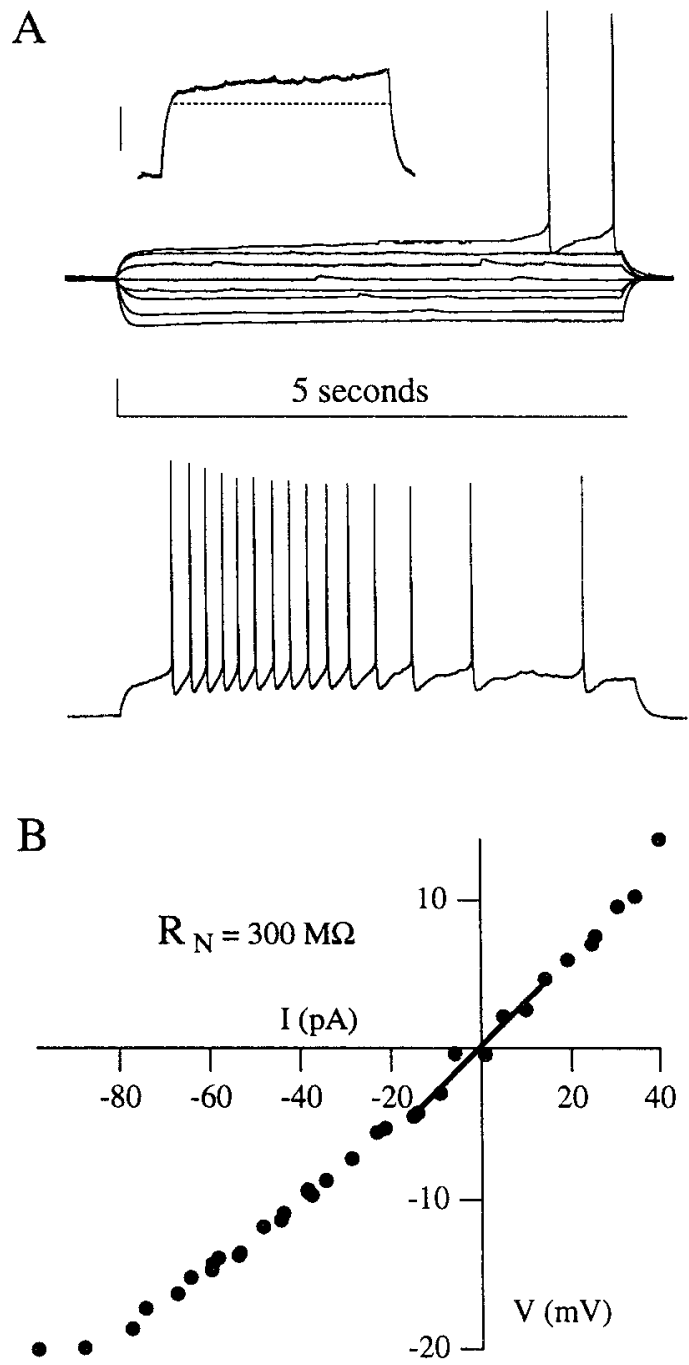

Figure 2. Physiological properties of late-spiking neurons. A, Voltage traces evoked by $5 \mathrm{sec}$ depolarizing and hyperpolarizing somatic current steps from resting potential. The top traces indicate subthreshold and just-suprathreshold voltage responses, whereas the bottom trace illustrates a response to a current step well above threshold. Note the ramp depolarizations and long delays before spike train onset that are typical of LS cells. A subthreshold ramp depolarization in response to a $5 \mathrm{sec}$ current step is shown in the inset (calibration in inset, $10 \mathrm{mV}$; the dashed line is a horizontal line for comparison). $B$, Current-voltage relations for this neuron, including the linear fit used to compute the input resistance of each neuron, $R_{\mathrm{N}}$. This cell had a pyramidal morphology (pictured in Fig. $5 A)$ and showed strong spike frequency adaptation across its spike train. The main calibration in $A: 20 \mathrm{mV}, 5 \mathrm{sec}$. 


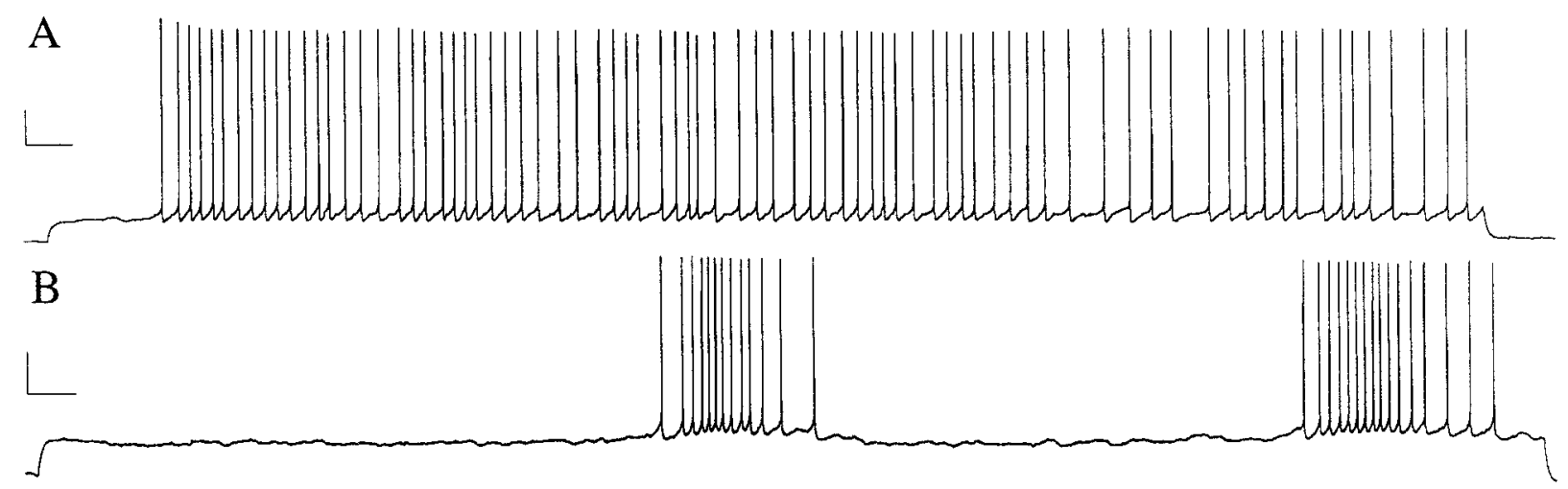

Figure 3. Responses of late-spiking neurons to very long depolarizing current steps. $A$, In response to a 60 sec depolarizing current step, this neuron delayed for $\sim 10 \mathrm{sec}$ and then began a spike train that was sustained for the duration of the step. $B$, In contrast to $A$, this neuron responded to a 60 sec step with a $24 \mathrm{sec}$ delay, followed by groups of spikes separated by long intervals. Calibration: $20 \mathrm{mV}, 2 \mathrm{sec}$.

Visual selection of neurons. We observed a wide variety of cellular morphologies under video microscopy, and we deliberately tried to include the full range of morphologies in our recordings in the hope of observing all of the cell types found in layer VI. Cells also were selected on the basis of visual indications of cell health, as described previously (Moyer and Brown, 1998). It is worth noting that one particular cellular morphology was easily distinguishable from the other cell types because of its small, round cell body without thick dendrites (Fig. 1, small cell near the calibration bar). On the basis of our previous work in PR, we know that this morphology is usually characteristic of fast-spiking (FS) cells (Faulkner and Brown, 1999). To represent the frequency of these small cells appropriately in our data set, we deliberately recorded from four of them ( $\sim 5 \%$ of our data set), which corresponds to their frequency (5-7\%) in a Golgi-Cox study of layer V I of PR in both juvenile and adult rats (our unpublished observations).

Data analysis. All voltage measurements were corrected for a $+13 \mathrm{mV}$ liquid junction potential between the bath and patch pipette solution (Moyer and Brown, 1998). Data were analyzed off-line, using customwritten software for IgorPro. Current-voltage $(I-V)$ relations were constructed for each neuron. Neuronal input resistance $\left(R_{\mathrm{N}}\right)$ was calculated from the slope of the line (least-squares regression) in the linear portion of the $I-V$ relationship, near the resting potential, where alternating hyperpolarizing and depolarizing voltage responses were symmetrical. Measurements of $R_{\mathrm{N}}$ were made well after voltage response had reached steady state, typically $100 \mathrm{msec}$ before the end of the current step. The time constant of the membrane voltage response $\left(\tau_{\mathrm{m}}\right)$ was estimated by an exponential fit to the voltage excursion in response to a small (1-10 pA) current step or, in a subset of cells, an average of 5-15 such responses. This averaging sometimes was required because of spontaneous synaptic activity. Action potential amplitude was measured from spike threshold. The latency of the first spike was measured relative to current step onset on the smallest supra-threshold current step. Statistical analyses were performed with StatView (Abacus Concepts, Calabasas, CA). All statistical comparisons between cell types use the nonparametric Mann-Whitney $U$ test (partly because of the greatly unequal sample sizes).
Histology and serial reconstructions. Our methods for visualizing and reconstructing biocytin-filled neurons have been reported elsewhere (Faulkner and Brown, 1999). Briefly, fixed slices were subsectioned to a thickness of $50-65 \mu \mathrm{m}$ on a freezing microtome, neurons were visualized by using a DAB-intensified avidin-biotin-horseradish peroxidase reaction, and slices were fixed in glycerol. Each section was traced by using a camera lucida with a $40 \times$ or $63 \times$ objective, and neurons were reconstructed via alignment of serial sections. We reconstructed 59 of the 84 neurons that were filled with biocytin.

\section{RESULTS}

\section{Overview of neuronal types}

Of the 86 neurons we recorded in layer VI, all but one could be classified easily according to our existing system (Faulkner and Brown, 1999; Beggs et al., 2000), which includes five basic categories: late-spiking (LS), single-spiking (SS), fast-spiking (FS), burst-spiking (BS), and regular-spiking (RS) neurons (Faulkner and Brown, 1999). LS cells show a slow ramp depolarization before the onset of their spike trains (Fig. $2 A$ ). The ramp can last many seconds for near-threshold current steps, and the spike train can also last for many seconds (Fig. $3 A$ ). In contrast to LS cells, SS cells tend to fire a single early spike ( $\sim 40 \mathrm{msec}$ latency) in response to large current steps and cannot be induced to fire trains (see Fig. 6A). Fast-spiking (FS) cells show long-lasting trains of fairly rapid firing $(20-30 \mathrm{~Hz})$ with little sign of spike frequency adaptation in response to depolarizing current steps (see Fig. 7A). RS cells typically begin their spike train within 100 msec of current step onset and exhibit variable degrees of spike frequency adaptation (Faulkner and Brown, 1999; Beggs et al., 2000). BS cells are seen only in layer V (Faulkner and Brown, 1999; Beggs et al., 2000) and are not relevant to the present study.

\begin{tabular}{lccc}
\hline \multicolumn{2}{l}{ Table 1. Properties of neurons in layer VI of juvenile perirhinal cortex } \\
& Late-spiking (67) & Single-spiking (5) & Fast-spiking (3) \\
\hline Resting membrane potential $(\mathrm{mV})$ & $-80.9 \pm 0.6$ & $-81.1 \pm 2.4$ & $-80.0 \pm 1.3$ \\
Input resistance $(\mathrm{M} \Omega$ ) & $557 \pm 36$ & $577 \pm 273$ & $269 \pm 70$ \\
Latency to first spike at threshold (msec) & $3165 \pm 140^{*}$ & $81 \pm 29^{*}$ & $79.3 \pm 29.1$ \\
Time constant $(\mathrm{msec})$ & $102.3 \pm 4.0 \dagger$ & $70.7 \pm 17.6 \dagger$ & $37.5 \pm 13.7$ \\
Spike threshold (mV) & $-53.0 \pm .4$ & $-54.7 \pm 4.4$ & $-59.3 \pm 1.5$ \\
Action potential amplitude (mV) & $86.1 \pm 1.3 \dagger$ & $74.7 \pm 4.2 \dagger$ & $63.4 \pm 4.5$ \\
Action potential overshoot $(\mathrm{mV})$ & $46.2 \pm 1.2 \dagger$ & $33.0 \pm 4.4 \dagger$ & $17.4 \pm 4.9$
\end{tabular}

*Indicates a statistically significant difference between LS and SS; $p<0.001$.

$\dagger$ Indicates a statistically significant difference between LS and SS; $p<0.05$.

FS cells were not compared statistically with the other types because of their small sample size. 
Figure 4. Serial reconstructions of biocytinfilled late-spiking neurons. The dashed line next to each neuron represents the near edge of the external capsule $(e c)$, except in $C$, where the near and far edges of the $e c$ are marked, with the lateral nucleus of the amygdala $(A L a)$ on the far side. Axons are omitted for clarity, and spines (present on $A$ and $D$ ) are representative. Scale bar, $100 \mu \mathrm{m}$.
A

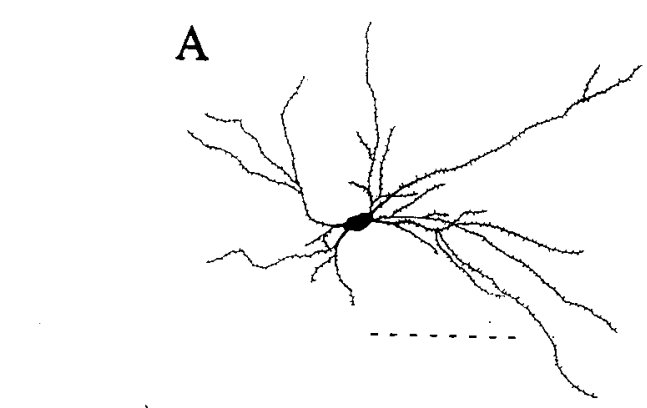

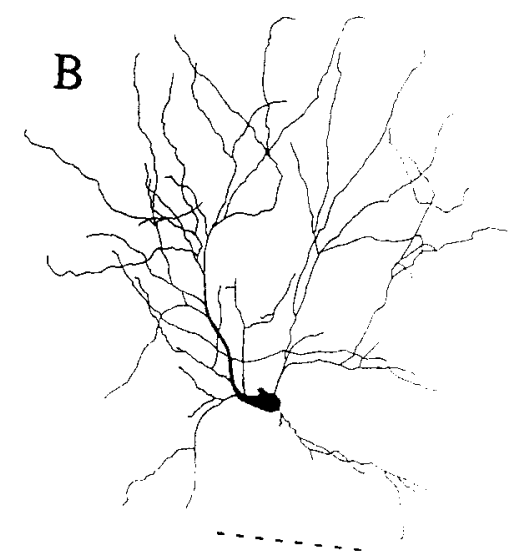

There were three startling findings. First, a vast majority (74 of 86 , or $86 \%$ ) of the neurons we recorded in PR layer VI were LS cells. This contrasts starkly with any layer of any cortex studied to date. Second, SS neurons were the next most common type (6 of 86 , or $7 \%$ ). SS cells have been reported in the amygdala, but never before in cortex. Third, only one cell (1 of 86 , or $1 \%$ ) behaved like the ubiquitous cortical RS neuron, which is the most common cell type in most cortical regions (McCormick et al., 1985; Connors and Gutnick, 1990). There was also one expected finding. Recall that we included four recordings from cells that looked like FS neurons on the basis of our observations in other layers of PR. The results verified in layer VI our finding in other layers of PR that we can identify these FS neurons visually before recording. Based on the IR-DIC images, these small cells are present but not numerous in layer VI of PR, consistent with our preliminary Golgi-Cox studies of the region (our unpublished observations).

\section{Late-spiking cells}

\section{Subthreshold properties}

The subthreshold membrane properties of LS and SS cells from juvenile rats are presented in Table 1 . The LS neurons typically had a resting potential at approximately $-81 \mathrm{mV}$, a high input resistance $\left(R_{\mathrm{N}}\right.$, usually in excess of $\left.500 \mathrm{M} \Omega\right)$, and a long time constant $\left(\tau_{\mathrm{m}}\right)$ associated with the membrane voltage response to a current step (but see data from adult animals, below). The $I-V$ relation for a typical LS cell is shown in Figure 2.

\section{Suprathreshold properties}

In response to a just-suprathreshold depolarizing current step, LS cells show a several second ramp depolarization, terminating in

one or more action potentials, depending on the duration of the current step. The latency to the first spike was determined with a just-suprathreshold, 5-sec-long step. The mean latency we recorded in neurons from juvenile animals was $3.2 \pm 0.1 \mathrm{sec}$. Additional suprathreshold membrane properties are reported in Table 1. LS neurons typically showed very slow afterhyperpolarizations (AHPs) after each spike in their train, which became briefer with larger current steps. We observed varied patterns of spike frequency adaptation among LS cells, ranging from cells that exhibited almost no adaptation to cells that ceased firing well before the end of the $5 \mathrm{sec}$ current step. In addition, a subset of cells showed reliable pauses in their spike trains. As reported previously in PR (Faulkner and Brown, 1999; Beggs et al., 2000), some LS cells showed a gradual acceleration of firing rate, or anti-adaptation, early in their spike train.

In many LS neurons the subthreshold ramp depolarization did not appear to reach steady state before the end of our standard 5 sec current step. Therefore, in a small subset of cells we also delivered much longer current steps $(10-60 \mathrm{sec})$ to evaluate the delay to the first spike. With these longer steps some neurons exhibited delays of 10-15 sec between the onset of the current step and the beginning of the spike train. When using these longer current steps, we also observed differences in the firing patterns among cells. Some neurons sustained their firing for the duration of the step (Fig. $3 A$ ), whereas others exhibited clusters of spikes several seconds long and separated by intervals of several seconds (Fig. 3B). These experiments seemed to have an adverse effect on cell health, so we did not give these very long current steps routinely. As a result, we did not correlate length of maximal delay 

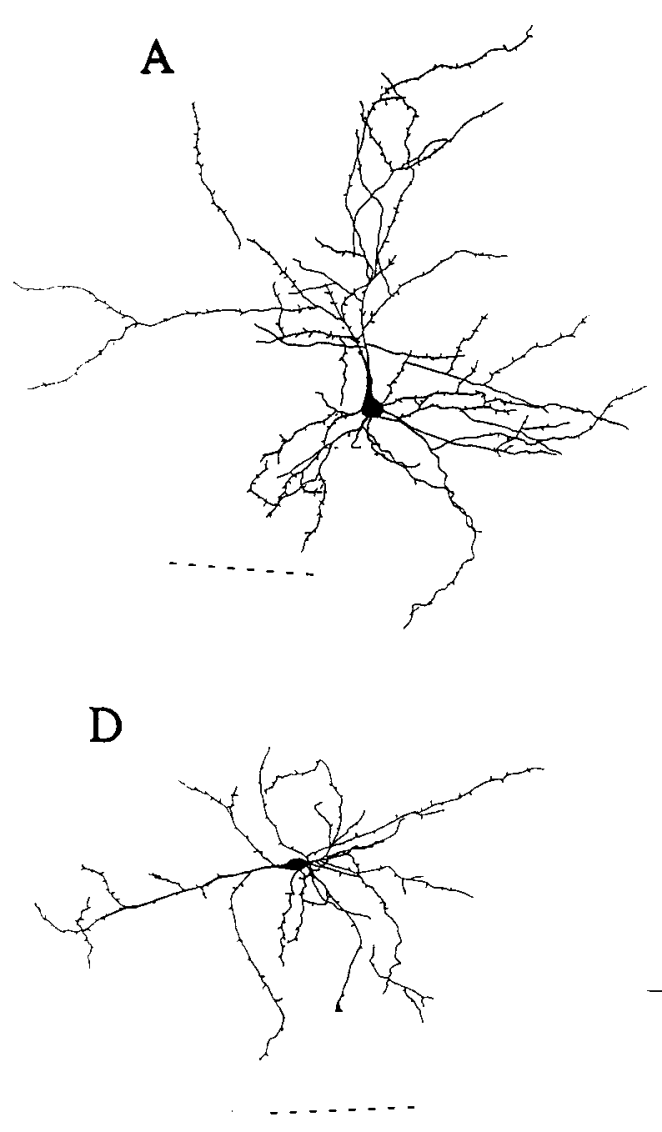

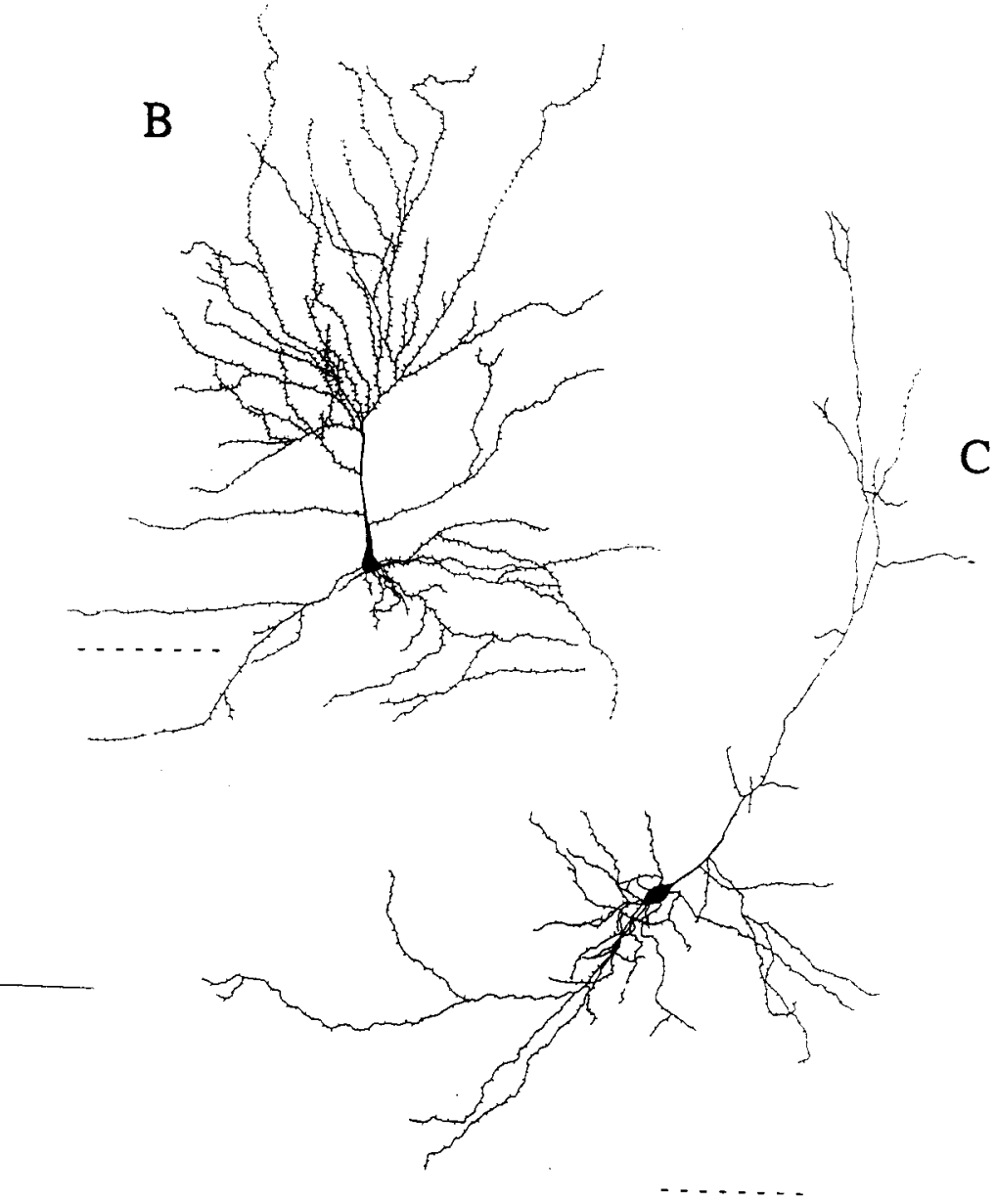

Figure 5. Serial reconstruction of biocytin-filled spiny pyramids in layer VI. All four of the illustrated neurons are late-spiking cells. The dashed lines indicate the near edge of the external capsule; axons are omitted for clarity, and spines are representative. Scale bar, $100 \mu \mathrm{m}$.

or long-term firing pattern with other physiological or morphological properties.

\section{Morphology}

Biocytin cell fills revealed a wide range of cellular morphologies among LS neurons, including both pyramidal and nonpyramidal cells. Filled neurons were most frequently nonpyramidal, with ovoid cell bodies and spiny, bitufted dendritic trees (Fig. 4A,D). However, similar neurons were observed without spines (Fig. $4 C$ ). Many of the filled cells were of the spiny modified pyramidal cell type common in layer V I of the cerebral cortex (Peters and Jones, 1984; Tömböl, 1984), as pictured in Figure 5. As observed in other cortical regions (Tömböl, 1984), a subset of these were oriented horizontally (Fig. 5D), and some were aspiny (Fig. 4B). Our biocytin staining did not label the full axonal arborization reliably, although previous work has revealed some projections into layers V and II/III (Faulkner and Brown, 1999). We found that the dendrites and axons (when visible) of LS neurons sometimes spanned the external capsule, passing into the lateral nucleus of the amygdala (ALa).

\section{Single-spiking cells}

We recorded a small number (6 of 86 , or $7 \%$ ) of single-spiking cells, which could be driven to fire only one spike, typically $\sim 40$ msec after current step onset (Fig. 6A). The mean latency of this first just-suprathreshold spike in SS cells was significantly different from that of LS cells $(p<0.001)$ in juvenile animals. SS cells often showed strong rectification in their $I-V$ relation (Fig. $6 C$ ). Their membrane properties were generally very similar to LS cells (Table 1), but SS neurons typically had smaller action potentials than LS neurons $(p<0.05)$ and shorter time constants $(p<0.05)$. We successfully reconstructed four biocytin-filled SS neurons, three of which were nonpyramidal (Fig. 6D) and one of which was pyramidal. We could not distinguish SS cells from LS cells morphologically in camera lucida reconstructions or under video microscopy in the brain slice.

\section{Fast-spiking cells}

We recorded from a few neurons with small round cell bodies, such as the bottom cell in Figure 1. As expected from our earlier studies of other layers of PR, we found these small, infrequent neurons to be FS cells (Fig. 7). Although they had a similar resting potential to the other cell types, FS cells had a lower input resistance and much shorter time constants $(\tau$; Table 1$)$. In addition, they exhibited a lower spike threshold than either LS or SS neurons and shorter action potentials with less overshoot. Note that statistical comparisons between FS cells and other cells types were not performed because of the small FS sample size. FS cells were capable of sustained firing at high rates $(30-70 \mathrm{~Hz})$, with spikes separated by very brief AHPs (Fig. $7 B$ ). The FS cells typically fired their first spike within $20-40 \mathrm{msec}$ of current step 

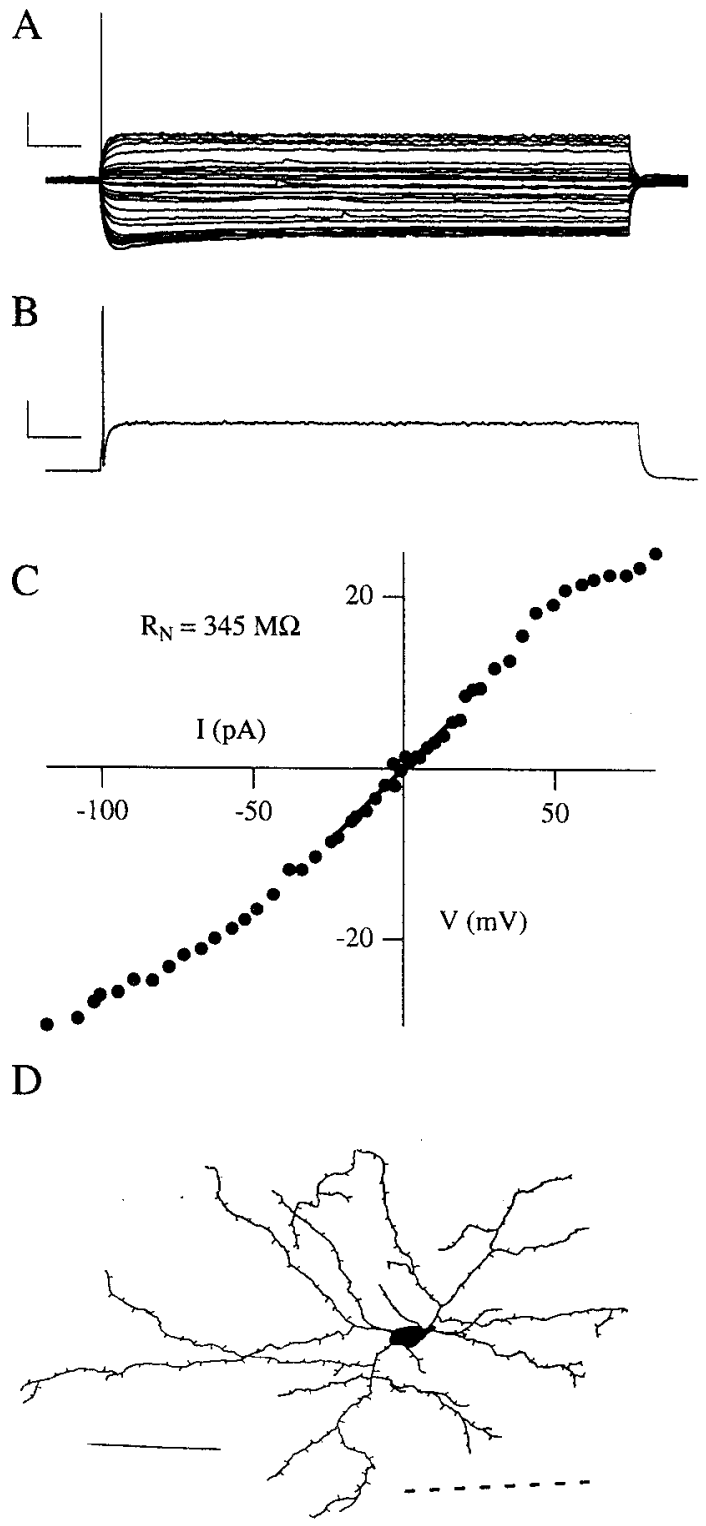

Figure 6. Physiological properties of single-spiking neurons. A, Voltage traces evoked by $5 \mathrm{sec}$ depolarizing and hyperpolarizing somatic current steps from resting potential. This set of traces indicates subthreshold and just-suprathreshold voltage responses. $B$, The voltage response to a current step well above threshold. Note that this cell (like other SS cells) fires only single early spikes, even with large current steps. Calibration in $A, B$ : $20 \mathrm{mV}, 500 \mathrm{msec}$. $C$, Current-voltage relations for this neuron, with the linear fit used to compute its input resistance, $R_{\mathrm{N}}$. Note the rectification in this $I-V$ plot. $D$, A serial reconstruction of a biocytin-filled SS cell, with spiny dendrites and a nonpyramidal morphology. The dashed line indicates the near edge of the external capsule. Scale bar, $100 \mu \mathrm{m}$.

onset, followed by the spike train. With small, just-suprathreshold current steps FS cells often showed "pauses" of 1 sec or more during their otherwise high-frequency spike trains (Fig. $7 A$ ), but large current steps could evoke prolonged firing for the duration of the step (Fig. 7B). These findings are comparable with our previous report of FS cells in PR (Faulkner and Brown, 1999).

We obtained morphological reconstructions from three FS neurons, one of which is illustrated in Figure 7D. All three FS neurons had small, very round cell bodies with relatively simple aspiny dendritic arborizations. The axon of the FS cell in Figure $7 D$ was filled well and projected locally within layer VI, consistent with the
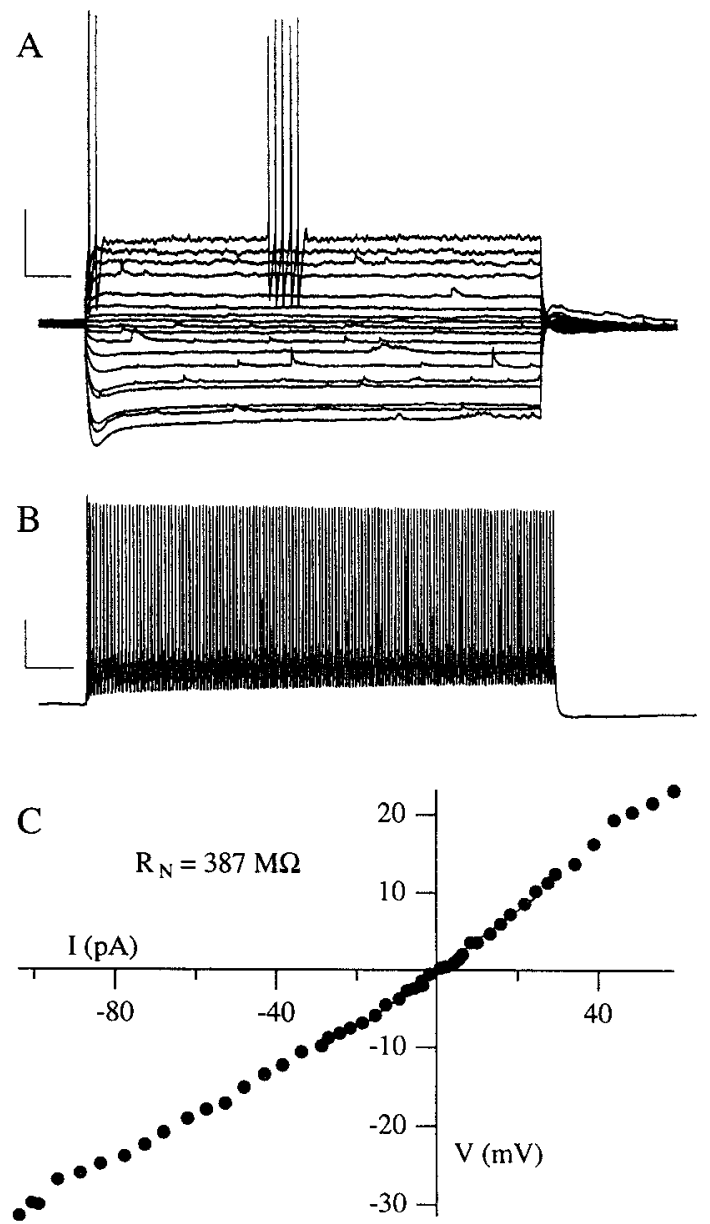

$\mathrm{D}$

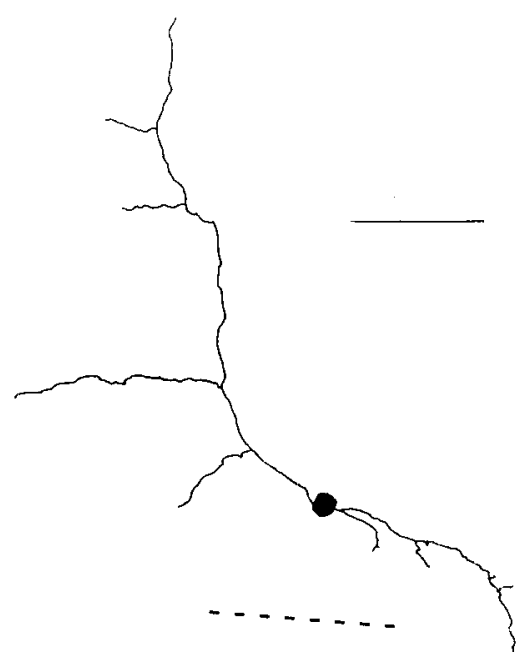

Figure 7. Physiological properties of fast-spiking neurons. A, Voltage traces evoked by $5 \mathrm{sec}$ depolarizing and hyperpolarizing somatic current steps from resting potential. This set of traces indicates subthreshold and just-suprathreshold voltage responses. $B$, The voltage response to a current step well above threshold. Note that this neuron has a short time constant and begins to fire spikes early in the current step. Moreover, it is capable of sustained discharge at high rates. This FS cell showed a pronounced sag in its hyperpolarizing voltage records. Calibration in $A, B: 20 \mathrm{mV}, 500 \mathrm{msec}$. $C$, Current-voltage relations for this neuron, with the linear fit used to compute its input resistance, $R_{\mathrm{N}}$. $D$, Serial reconstruction of this biocytinfilled FS cell, showing a simple aspiny dendritic arbor and small, round cell body. The axon of this cell (omitted for clarity) ramified locally. The dashed line indicates the near edge of the external capsule. Scale bar, $100 \mu \mathrm{m}$. 
Table 2. Properties of LS neurons in layer VI of adult perirhinal cortex $(n=7)$

Resting membrane potential (mV)

Input resistance $(\mathrm{M} \Omega)$

Latency to first spike at threshold (msec)

Time constant (msec)

Spike threshold $(\mathrm{mV})$

Action potential amplitude $(\mathrm{mV})$

Action potential overshoot $(\mathrm{mV})$

*Indicates a statistically significant difference between adult LS and juvenile LS; $p<$ 0.01 .

$\dagger$ Indicates a statistically significant difference between adult LS and juvenile LS; $p<$ 0.05 .

hypothesis that FS cells in PR are local interneurons (Faulkner and Brown, 1999). Morphologically and physiologically, these FS cells seem similar to those we see throughout PR (Faulkner and Brown, 1999).

\section{Data from adult rats}

We often record from brain slices from juvenile rats (13-20 d old) because these slices yield the healthiest neurons and the best visualization. However, the behavioral work on the role of PR has been conducted exclusively on adult animals, so it seemed important to establish whether the distribution of cell types in these juvenile rats resembles that in adults. We therefore recorded from nine neurons in four rats aged 3.5 months. The relative numbers of each type in recordings from the adult rats were similar to those from juvenile rats: seven of the nine neurons were late-spiking cells (78\%), one of the nine neurons was a single-spiking cell (11\%), and the neuron predicted to be a fast-spiking cell on the basis of its appearance under video microscopy was confirmed as a FS cell when it was patched. The membrane properties of the LS neurons recorded in slices from adult rats are presented in Table 2. Statistical comparison of adult and juvenile LS neurons revealed that the adult cells had lower input resistances $(p<0.01)$ and shorter time constants $(p<0.01)$. In recording from neurons from the adult rats, we used the same method for finding the latency of first spike at threshold as we did in the younger animals. Because of the reduced input resistance in adult neurons, the standard changes in current step amplitude that were used to find a just-suprathreshold step (see Materials and Methods) afforded a greater effective resolution in finding threshold in neurons from older animals. As a result, the measurements of first spike latency at threshold presumably were biased significantly toward longer measurements in adult neurons. For this reason we discounted the statistically significant latency difference. The small number of recordings of adult FS and SS neurons precluded meaningful comparison of their membrane properties with their juvenile equivalents. We successfully obtained morphological reconstructions from six of the nine adult neurons from which we recorded (five LS and one SS). As in the young animals, these cells included both pyramidal and nonpyramidal morphologies. However, several of the adult neurons had much larger and more elaborate dendritic arbors than were common among the neurons from juvenile rats (data not shown), which is consistent with other studies of cortical dendritic development (Petit et al., 1988; Zhu, 2000) and with the decreased input resistance in the neurons from adult rats.

\section{DISCUSSION}

One major finding of this study is that layer VI of perirhinal cortex is composed almost entirely of late-spiking neurons (74 of 86 , $\sim 86 \%$ ), with almost no ( 1 of $86, \sim 1 \%$ ) RS neurons and no BS neurons. Another significant finding is that layer VI also contains a notable concentration of single-spiking neurons (6 of 86, 7\%), which have not been reported previously in the cerebral cortex. The abundance of LS cells is particularly remarkable because we found them to vary greatly in morphology, including both pyramidal and nonpyramidal cells that could be spiny or aspiny. In addition, we observed that layer VI contains a small number of fast-spiking cells, which could be identified visually under IR-DIC microscopy.

In both layer VI and layer II/III (where LS neurons comprise $\sim 50 \%$ of the population of pyramidal cells; see Beggs et al., 2000) the abundance of LS neurons in PR dwarfs that found in any other part of the cerebral cortex, establishing that the cellular neurophysiology of PR is different from that of any other cortical region studied to date. LS cells also have been seen in rat frontal cortex (Kawaguchi, 1995) and neostriatum (Nisenbaum et al., 1994), although their reported delays generally have been well under 1 sec. In frontal cortex (the only other cortical region in which LS neurons have been seen) these cells are reported to be infrequent, comprising $\sim 11 \%$ (9 of 83 ) of the nonpyramidal neurons in layer II/III (Kawaguchi, 1995).

The abundance of LS neurons in layer VI is unlikely to be an artifact of our methodology for several reasons. First, we have used identical recording methods to survey each of the layers of PR and found the distribution of LS neurons to be layer-specific. In fact, we have found that the layer with the lowest concentration of LS cells, layer $\mathrm{V}$, actually is sandwiched between the two layers with high concentrations, layers II/III and VI. Second, it is unlikely that our visual preselection of neurons biased the results toward LS neurons because we deliberately tried to record from the full range of cellular morphologies discernible under video microscopy. Our cell fills indicate that we succeeded in recording from cells of widely varying morphology (see below). Third, the use of room temperature $\left(23-24^{\circ} \mathrm{C}\right)$ recording saline throughout most of this study is unlikely to produce late spiking because we found LS neurons in layer VI in a small number of experiments that were performed at $30-32^{\circ} \mathrm{C}$ (data not shown). Likewise, Nisenbaum and colleagues (1994) found that similar neurons in the striatum show late spiking both at $33^{\circ} \mathrm{C}$ and at $20-22^{\circ} \mathrm{C}$ (Gabel and Nisenbaum, 1998). It should be noted that a preliminary survey of PR from this laboratory reported seven RS cells in layer VI in a sample of 22 (Faulkner and Brown, 1999). This discrepancy with the current results probably was produced by the inherent difficulty of discerning the border between layer V and layer VI. The present study was more conservative, recording only from neurons that were clearly in layer VI and confirming the placement of most electrodes at low magnification during the recording.

\section{Mechanisms of late spiking}

As we look across the layers of PR, it is obvious that LS neurons can have any of a broad range of cellular morphologies. In layer VI, LS neurons are morphologically heterogeneous, including both pyramidal and nonpyramidal cells, whereas they are typically small pyramids in layer II/III and large pyramids in layer V. This variety seems to support the hypothesis that late spiking emerges from the selective expression of ionic conductances rather than dendritic structure per se (cf. Mainen and Sejnowski, 1996). We previously reported that LS neurons exhibit a slowly developing inward current when the membrane potential is stepped from rest to a just-subthreshold level (Faulkner and Brown, 1999). This current was hypothesized to underlie the 
delay and slow ramp depolarization exhibited by these neurons. Our preliminary results in both layer VI and layer II/III indicate that this current is blocked by low micromolar concentrations of 4-aminopyridine, which also blocks the ramp depolarization and delay (Moyer et al., 2000), suggesting that late spiking may be produced by a slowly inactivating potassium conductance. This conjecture is consistent with results from the striatum (Nisenbaum et al., 1994) and hippocampus (Storm, 1988). The large population of LS neurons in layer VI provides a convenient opportunity to study the molecular biology, ionic mechanisms, and modulation of LS neurons.

\section{Neuronal types in adult perirhinal cortex}

To our knowledge, this study is the first demonstration of latespiking cells and single-spiking cells in the cortex of adult rats. In combination with our recent observations in layer $\mathrm{V}$ of $\mathrm{PR}$ in adult rats (Moyer, McNay, and Brown, unpublished observations), which included regular-spiking and burst-spiking cell types not seen in layer VI, we have now seen all five of the major PR cell types in adult rats. Moreover, the data from adult rats presented here suggest that the gross proportions of the firing types remain similar (within sampling error) from the second postnatal week at least into adulthood in layer VI of PR.

\section{Relation of perirhinal cortex and lateral amygdala}

Swanson and Petrovich (1998) recently proposed that the lateral nucleus of the amygdala might be better considered as the deepest layer of the overlying cortex than as a part of the amygdala. Our findings support this idea in two ways. First, in our cell fills in layer VI of PR we found that neurons often sent their dendrites across the external capsule into ALa, and our data probably underrepresent this crossover because our fixed tissue tended to crack along the line of the capsule. Second, we encountered a small but substantial number of SS cells in PR layer VI, a cell type that has been reported previously in ALa (Chapman et al., 1990; Faulkner and Brown, 1999) and the nucleus tractus solitarii (Paton et al., 1993) but has never been reported before in cortex. This perirhina1-amygdala relationship could be involved in the emotional disturbances that often accompany perirhinal pathology.

\section{Uniqueness of perirhinal cortex}

Many investigators have sought an "elementary pattern of cortical organization" (Douglas and Martin, 1998) and tried to find underlying elements of "uniformity" (Mountcastle, 1998) amid the diversity of cortex. Such efforts are important, but there is also a need for comparative studies to evaluate whether the underlying principles of organization found in one cortical area can be generalized validly to other areas (Connors and Gutnick, 1990). The data presented here support our growing understanding that perirhinal cortex is very different from every other area of the cerebral cortex that has been studied to date, for it contains large numbers of very unusual cells although it is lacking entirely in certain fundamental classes of neurons in some layers. Further study of the unique cellular neurobiology of PR may provide new insight into the neuronal and circuit level mechanisms of information processing as well as clues to selective vulnerability to neurodegeneration.

\section{REFERENCES}

Beggs JM, Moyer JR, McGann JP, Brown TH (2000) Delayed synaptic integration in layer II/III pyramidal neurons of rat perirhinal cortex. J Neurophysiol 83:3294-3298.

Buffalo EA, Reber PJ, Squire LR (1998) The human perirhinal cortex and recognition memory. Hippocampus 8:330-339.

Burwell RD, Amaral DG (1998a) Cortical afferents of the perirhinal, postrhinal, and entorhinal cortices of the rat. J Comp Neurol 398:179-205.

Burwell RD, Amaral DG (1998b) Perirhinal and postrhinal cortices of the rat: interconnectivity and connections with the entorhinal cortex. J Comp Neurol 391:293-321.

Burwell RD, Witter MP, Amaral DG (1995) Perirhinal and postrhinal cortices of the rat: a review of the neuroanatomical literature and comparison with findings from the monkey brain. Hippocampus 5:390-408.

Chapman PF, Kairiss EW, Keenan CL, Brown TH (1990) Long-term synaptic potentiation in the amygdala. Synapse 6:271-278.

Colbert CM, Johnston D (1996) Axonal action potential initiation and $\mathrm{Na}^{+}$channel densities in the soma and axon initial segment of subicular pyramidal neurons. J Neurosci 16:6676-6686.

Connors BW, Gutnick MJ (1990) Intrinsic firing patterns of diverse neocortical neurons. Trends Neurosci 13:99-104.

Douglas R, Martin K (1998) Neocortex. In: The synaptic organization of the brain (Shepherd GM, ed), pp 459-509. New York: Oxford UP.

Falls WA, Bakken KT, Heldt SA (1997) Lesions of the perirhinal cortex interfere with conditioned excitation but not with conditioned inhibition of fear. Behav Neurosci 111:476-486.

Faulkner B, Brown TH (1999) Morphology and physiology of neurons in the rat perirhinal-lateral amygdala area. J Comp Neurol 411:613-642.

Gabel LA, Nisenbaum ES (1998) Biophysical characterization and functional consequences of a slowly inactivating potassium current in neostriatal neurons. J Neurophysiol 79:1989-2002.

Kawaguchi Y (1995) Physiological subgroups of nonpyramidal cells with specific morphological characteristics in layer II/III of rat frontal cortex. J Neurosci 15:2638-2655.

Mainen ZF, Sejnowski TJ (1996) Influence of dendritic structure on firing pattern in model neocortical neurons. Nature 382:363-366.

McCormick DA, Connors BW, Lighthall JW, Prince DA (1985) Comparative electrophysiology of pyramidal and sparsely spiny stellate neurons of the neocortex. J Neurophysiol 54:782-806.

McCreless MP, McGann JP, Brown TH (2000) Pavlovian conditioning interstimulus interval function emerges from synaptic noise and neuronal dynamics. Soc Neurosci Abstr 26:1505.

McGann JP, Brown TH (2000) Fear conditioning model predicts key temporal aspects of conditioned response production. Psychobiology 28:303-313.

Meunier M, Bachevalier J, Mishkin M, Murray EA (1993) Effects on visual recognition of combined and separate ablations of the entorhinal and perirhinal cortex in rhesus monkeys. J Neurosci 13:5418-5432.

Mountcastle VB (1998) Perceptual neuroscience: the cerebral cortex. Cambridge, MA: Harvard UP.

Moyer Jr JR, Brown TH (1998) Methods for whole-cell recording from visually preselected neurons of perirhinal cortex in brain slices from young and aging rats. J Neurosci Methods 86:35-54.

Moyer Jr JR, McGann JP, Brown TH (2000) Analysis of late spiking in rat perirhinal cortex. Soc Neurosci Abstr 26:1628.

Mumby DG, Pinel JP (1994) Rhinal cortex lesions and object recognition in rats. Behav Neurosci 108:11-18.

Nisenbaum ES, Xu ZC, Wilson CJ (1994) Contribution of a slowly inactivating potassium current to the transition to firing of neostriatal spiny projection neurons. J Neurophysiol 71:1174-1189.

Otto T, Eichenbaum H (1992) Complementary roles of the orbital prefrontal cortex and the perirhinal-entorhinal cortices in an odor-guided delayed nonmatching-to-sample task. Behav Neurosci 106:762-775.

Paton JF, Foster WR, Schwaber JS (1993) Characteristic firing behavior of cell types in the cardiorespiratory region of the nucleus tractus solitarii of the rat. Brain Res 604:112-125.

Paxinos G, Watson C (1998) The rat brain in stereotaxic coordinates, 4th Ed. San Diego: Academic.

Peters A, Jones EG (1984) Classification of cortical neurons. In: Cellular components of the cerebral cortex (Peters A, Jones EG, eds), pp 107122. New York: Plenum.

Petit TL, LeBoutillier JC, Gregorio A, Libstug H (1988) The pattern of dendritic development in the cerebral cortex of the rat. Brain Res 469:209-219.

Storm JF (1988) Temporal integration by a slowly inactivating $\mathrm{K}^{+}$current in hippocampal neurons. Nature 336:379-381.

Suzuki WA (1996) The anatomy, physiology, and functions of the perirhinal cortex. Curr Opin Neurobiol 6:179-186.

Swanson LW, Petrovich GD (1998) What is the amygdala? Trends Neurosci 21:323-331

Tieu KH, Keidel AL, McGann JP, Faulkner B, Brown TH (1999) Perirhinal amygdala circuit-level computational model of temporal encoding in fear conditioning. Psychobiology 27:1-25.

Tömböl T (1984) Layer VI cells. In: Cellular components of the cerebral cortex (Peters A, Jones EG, eds), pp 479-520. New York: Plenum.

Wiig KA, Burwell RD (1998) Memory impairment on a delayed nonmatching-to-position task after lesions of the perirhinal cortex in the rat. Behav Neurosci 112:827-838.

Zhu JJ (2000) Maturation of layer 5 neocortical pyramidal neurons: amplifying salient layer 1 and layer 4 inputs by $\mathrm{Ca}^{2+}$ action potentials in adult rat tuft dendrites. J Physiol (Lond) 526:571-587. 